\title{
Perfil del egresado de la Licenciatura en Flauta de la Universidad Nacional de Cuyo y su inserción laboral:
}

\author{
la relación entre las herramientas brindadas en el plan \\ de estudios y la realidad del campo profesional \\ Juan Pablo Ganem Ábrego \\ [Facultad de Artes y Diseño - Universidad Nacional de Cuyo]
}

Resumen El presente artículo analiza el perfil del egresado de la Licenciatura en Flauta de la UNCuyo y su inserción laboral. Se focaliza en la relación entre las herramientas brindadas en los planes de estudio y las exigencias de un puesto de trabajo con especificidad en el campo del título obtenido, observada desde el punto de vista de un grupo de egresados y estudiantes avanzados de la carrera. Se utilizó una metodología mixta, articulando datos cuantitativos obtenidos a través de una encuesta de preguntas cerradas, con datos cualitativos construidos a partir de entrevistas. Sumado a un relevamiento de puestos de trabajo afines, que permitió tipificar la realidad laboral actual del flautista profesional en la Argentina, se describieron las dificultades que se les presentan a los egresados para lograr una carrera deseable y sustentable en el terreno de la performance musical. Por ello, es que se llegó a la conclusión de que, en un medio caracterizado por la escasez de puestos de trabajo especíicos, los egresados deben valerse de herramientas que trascienden a las ofrecidas en una formación de grado universitario.

Palabras clave: Flauta · Formación académica $\cdot$ Inserción laboral
Summary The present article that analyses the graduate's profile of the Bachelor's Degree in Flute of the National University of Cuyo and their labor insertion. Focuses on the connection between the tools offered in the study programmes and the demands of a job with specificity in the field of the obtained degree, analyzed from the point of view of graduates and advanced students of the course of study. A mixed methodology was used, where quantitative data, obtained through a survey of closedended questions, were articulated with qualitative data, obtained from interviews. Considering a gathering of jobs related to the degree object of this research, which allowed to typify the current Argentine working conditions of the professional flute player, it was possible to describe the difficulties that graduates face in achieving a desirable and sustainable career in the field of musical performance. For this reason, it was concluded that, in an environment characterized by the shortage of specific jobs, graduates must employ tools that go beyond those that can offer their undergraduate university education.

Keywords: Flute $\cdot$ Academic training Occupational insertion 


\section{1 - INVESTIGACIONES PREVIAS / PROBLEMA DE INVESTIGACIÓN}

Partimos del relevamiento de investigaciones con temáticas afines, de las cuales se mencionarán solamente cuatro: el libro La música clásica como profesión (2010) de la investigadora australiana Dawn Bennett, que analiza la música clásica como profesión a lo largo del tiempo y el cambio en las exigencias laborales. La inserción laboral de los estudiantes de música (2008) del español Alejandro Bújez, que se enfoca en las expectativas de los estudiantes de música y sus reales posibilidades de inserción laboral.

Bajo la misma línea de Bújez, se encuentra la investigación Formación Profesional e Inserción Laboral en Artes, Diseño y Humanidades (2014) del mendocino Roberto Tomassiello, un trabajo que se realizó en la FADUNCuyo sobre la inserción laboral de todas las carreras comprendidas dentro de esta facultad. Y, por último, más próximo a la presente investigación, el trabajo de Alejandra García Trabucco y Cristina Cuitiño, Formación Profesional e inserción laboral de los egresados de carreras musicales, FAD-UNCuyo, el cual abordó la problemática de la formación profesional y la inserción laboral de los egresados de las Carreras Musicales, FAD, UNCuyo.

Tras este recorrido bibliográfico, nos surgieron interrogantes que resultaron disparadores para esta investigación, los cuales pueden sintetizarse en la siguiente: ¿los graduados y estudiantes avanzados de la Licenciatura en Flauta (FAD-UNCuyo) lograron obtener, a lo largo de la carrera, las herramientas requeridas para aspirar a trabajar en la actividad que desean, acorde con la mencionada titulación, y poder hacer de ello un medio de vida?

Vale destacar que, de acuerdo con la información brindada por la página web de la FAD-UNCuyo y en el plan de estudios, el ser Licenciado en Flauta permite interpretar en el instrumento diversos géneros y estilos musicales, planificar, conducir y supervisar proyectos musicales, y realizar estudios, investigaciones y asesoramientos relacionados a su ámbito profesional específico. Ante esto, la pregunta que surge es ¿qué peso se le otorga a cada elemento que configuran el perfil del licenciado en flauta 
en la formación académica real, y cómo esto se relaciona con las posibilidades de inserción laboral?

Mi doble rol de investigador y miembro del colectivo investigado, aportó inevitablemente un conocimiento previo que me comprometí a confrontar con las evidencias que se recabaron. Al decir de la filósofa argentina Esther Díaz (2007), el investigador no es «ascético» sino que siempre forma parte del proceso hermenéutico, pero debe estar atento a sus preconceptos, para «ampliar su mirada y buscar sentidos que reconstruyan las motivaciones de lo analizado" (Díaz, 2007: 85).

La problemática compleja y de multicausalidad obliga a considerar no solo el perfil académico de la carrera reflejado en el plan de estudios, sino también los recorridos que realizan los estudiantes dentro de ese marco, dominados por sus expectativas y los aspectos que ellos mismos jerarquizan en sus años de estudio. Finalmente, es esa combinación de variables y las que aporta el cambiante medio laboral, lo que pesará al momento de insertarse profesionalmente.

\section{$2 \cdot$ Овjetivos}

\section{1 - Objetivo General}

Caracterizar la relación entre la formación profesional y la inserción laboral del egresado de la Licenciatura en Flauta de Carreras Musicales, FAD, UNCuyo, según la percepción de los egresados del período comprendido entre 2008-2019, y estudiantes avanzados actuales.

\subsection{Objetivos Especificos}

Realizar un diagnóstico de la realidad laboral actual del flautista profesional en la Argentina, a partir de los datos obtenidos a través del trabajo de campo. 
Describir las dificultades que se les presentan a los egresados para lograr una carrera deseable y sustentable en el terreno musical.

Observar las diferencias y similitudes de formación académica entre los egresados de la Licenciatura de Flauta, que cursaron con el Plan de estudio Ord. No: 29/99-Cs y los que lo hicieron con el Plan de estudio Ord. No: 84/ıO-Cs, y determinar si dicho cambio influyó en su inserción laboral.

Describir la trayectoria laboral de la persona que egresa hasta su inserción en un medio laboral deseado, en términos de las actividades que realiza en ese período intermedio.

\section{$3 \cdot$ Metodología}

Se utilizó una metodología mixta, donde se articularon datos cuantitativos obtenidos a través de una encuesta de preguntas cerradas, con datos cualitativos relevados a partir de entrevistas a tres flautistas profesionales que han desarrollado carrera de solistas, y a la Prof. Beatriz Plana, Titular de la Cátedra de Flauta (asignatura troncal de la Licenciatura) y, a su vez, destacada solista.

Por otro lado, con el fin de realizar un diagnóstico de la realidad laboral actual del flautista profesional en la Argentina, se realizó un relevamiento de las fuentes de trabajo estables y dependientes de algún organismo, que se vinculen con las competencias y aptitudes que los egresados y estudiantes consideren que posee un Licenciado en Flauta, de la FAD, UNCuyo. El mismo se hizo a través de un método cuantitativo denominado observación estructurada.

A su vez, se realizó un análisis comparativo, a través de una observación estructurada, de los planes de estudio que estuvieron en vigencia en el período estudiado. El mismo, tuvo el fin de determinar similitu- 
des y diferencias, así como también observar si dicho cambio fue favorecedor para la inserción laboral de los licenciados en flauta.

\section{$4 \cdot$ Desarrollo}

\subsection{Encuesta a egresados y estudiantes avanzados}

Como se mencionó anteriormente, se construyó una encuesta con preguntas cerradas y, como el propósito de la misma no fue dar a conocer el desempeño laboral personal de cada participante, sino poder hacer un relevamiento de la realidad laboral de los egresados y estudiantes en general, se decidió hacerla anónimamente. Estimamos que el factor de anonimato resultó beneficioso al momento de emitir las respuestas, ya que, de las veintidós personas a las que se les envío la encuesta, dieciocho la contestaron. Dicha encuesta, se organizó en 5 ejes.

\subsubsection{Datos sociodemográficos}

Los encuestados tenían un rango de edad que variaba desde los 2I a los 38 ańos, la mitad de ellos eran egresados y la otra mitad estudiantes avanzados. De igual manera, la mitad de la presente población estudió con el Plan de Estudios Ord. $N^{\circ}$ : 29/99-CS (plan «viejo») y la otra mitad con el Plan Ord. $N^{\circ}: 84 /$ Io-cs (plan «nuevo»).

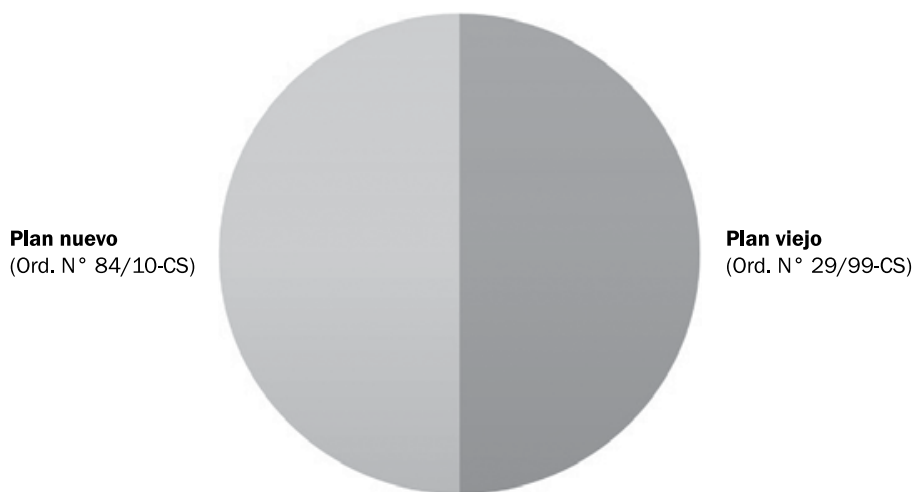

Gráfico I: Plan de estudios con el que el/la encuestado/a estudió. 
En cuanto a sus expectativas laborales al momento de ingresar a estudiar la Licenciatura en Flauta, vimos que la mayoría tuvo la expectativa de desenvolverse laboralmente como músico de orquesta (6I,I\%), dejando como segundo lugar músico de ensambles $(50 \%)$ y en tercer lugar intérprete solista $(38,9 \%)$. Es decir, expectativas vinculadas directamente con la labor de músico intérprete, siendo menor cantidad aquellos que seleccionaron actividades como lo son: la docencia, la gestión de proyectos musicales y la investigación.

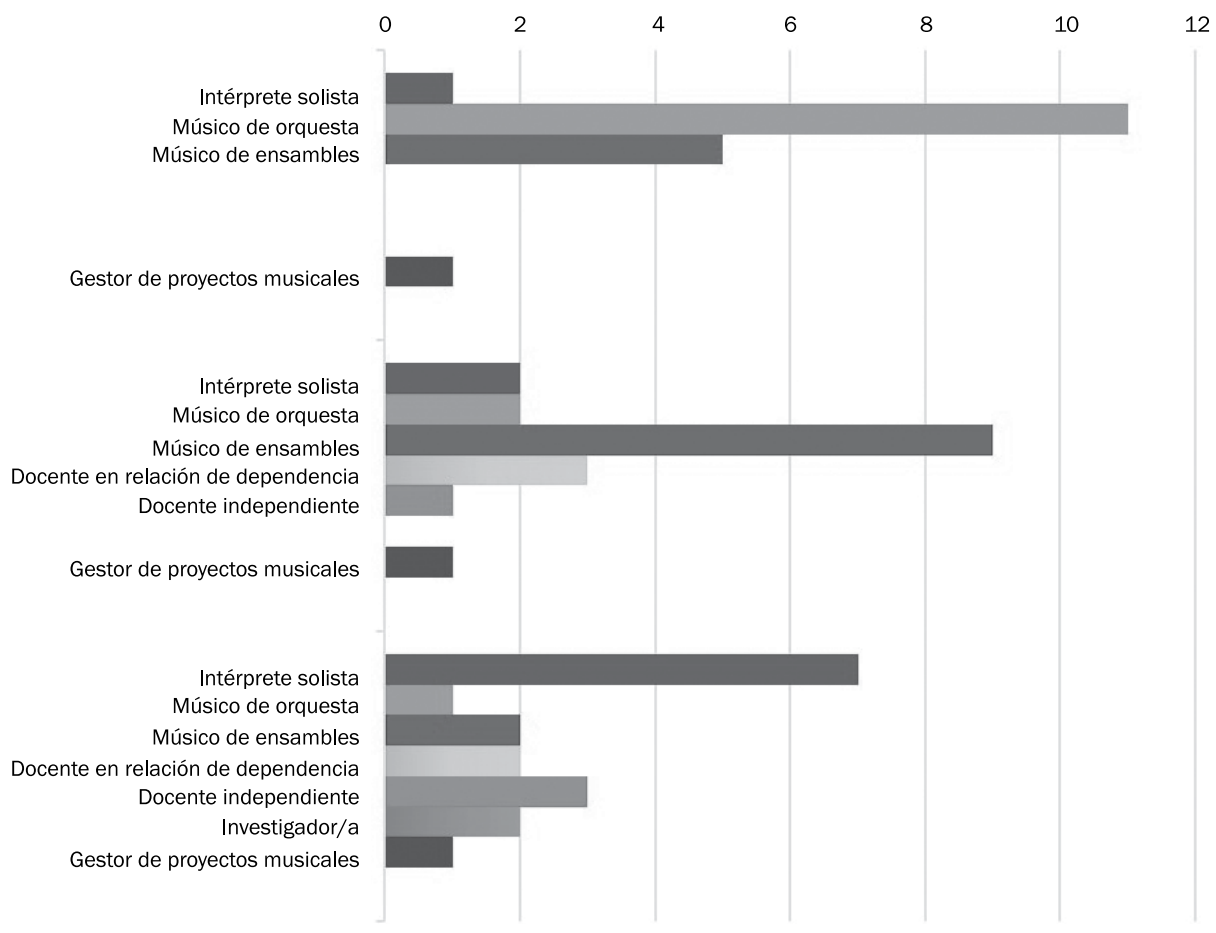

Gráfico 2: Expectativas laborales al momento de ingresar a la carrera 


\subsection{2 $\cdot$ Trabajo estable}

Se buscó conocer las percepciones que se tiene del trabajo estable. De acuerdo con los resultados obtenidos, los encuestados consideran que un trabajo es estable cuando se tiene un sueldo mensual garantizado, se cuenta con una obra social, vacaciones pagas, aportes previsionales, licencias por diferentes motivos, y, además, el trabajador puede planificar su vida como desee, en ese orden.

En segundo lugar, se los interrogó sobre las mejores estrategias para conseguir un empleo estable como flautista, seleccionando la experiencia laboral, la buena formación académica de grado y posgrado, cursos de perfeccionamiento y contactos.

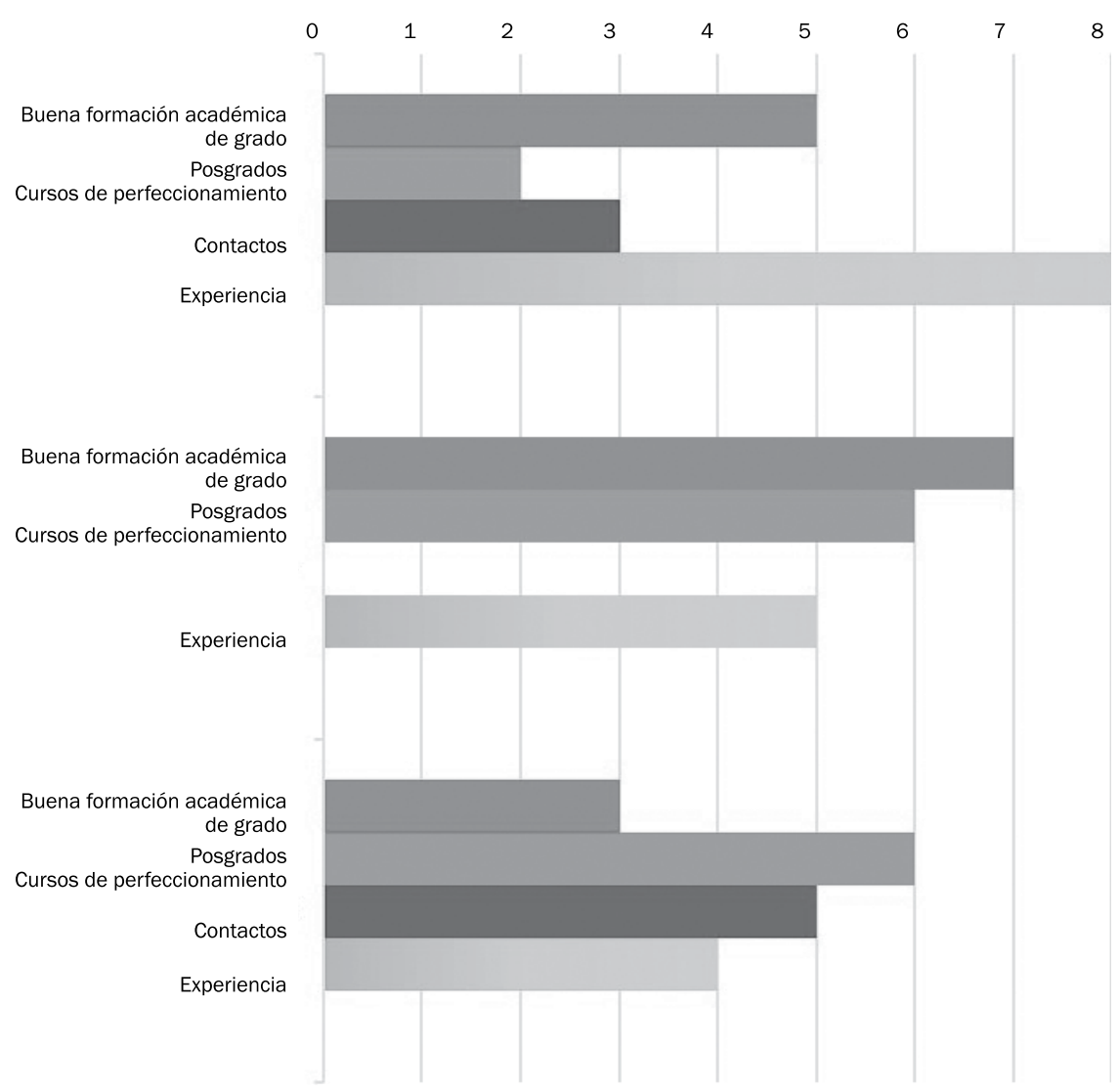

Gráfico 3: Las mejores estrategias para conseguir un empleo estable como flautista 
Por último, se indagó sobre los principales obstáculos para obtener un empleo estable como flautista, los cuales son: la escasa cantidad de puestos, favoritismo que se traduce en corrupción, la formación insuficiente, falta de experiencia laboral, título sin incumbencia (relacionado con la docencia). Tal como Bennett (20I0) sostiene, a lo largo de la historia, la problemática laboral del músico radica en que hay más personas que cargos a cubrir, se observa que el $77,78 \%$ de los encuestados concuerda con la autora.

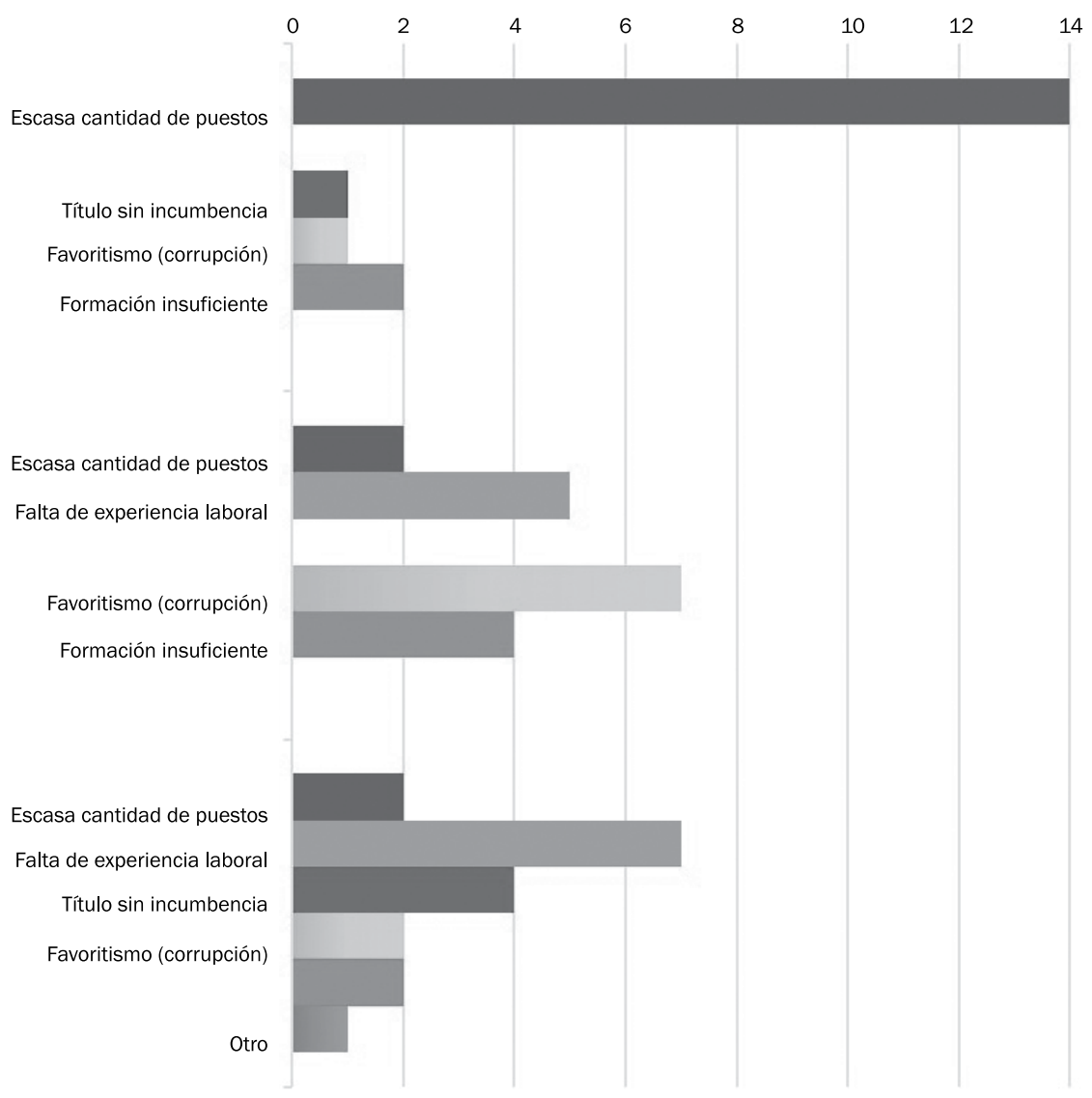

Gráfico 4: Los principales obstáculos para obtener un trabajo como flautista 


\subsection{3 - Trayectoria laboral}

Ante la primera pregunta: ¿Trabaja/ba mientras estudia/ba? Casi el $89 \%$ de los encuestados contestó que "sí», lo que muestra que, en general, se desarrolla una trayectoria laboral previa al egreso. Recordemos que esto es posible porque, en las carreras de performance artística, no es necesario contar con una matrícula habilitante para ejercer, como por ejemplo en las carreras de Medicina o Abogacía. Pero cabe mencionar que estos trabajos no siempre están relacionados con la interpretación de la flauta.

Podemos citar el ejemplo del ingreso a un puesto, estable o eventual, dentro de una Orquesta Sinfónica, y para ello nos apoyamos en la investigación llevada a cabo por la profesora argentina Nancy Diez (200920I4) quien, tras investigar sobre los criterios de selección de músicos para integrar una orquesta, concluyó que «el nivel de ejecución del instrumento es considerado como un criterio determinante para la aprobación de un concurso» mientras que «la formación académica no cuenta con gran relevancia dentro de los criterios de selección». (p. 170)

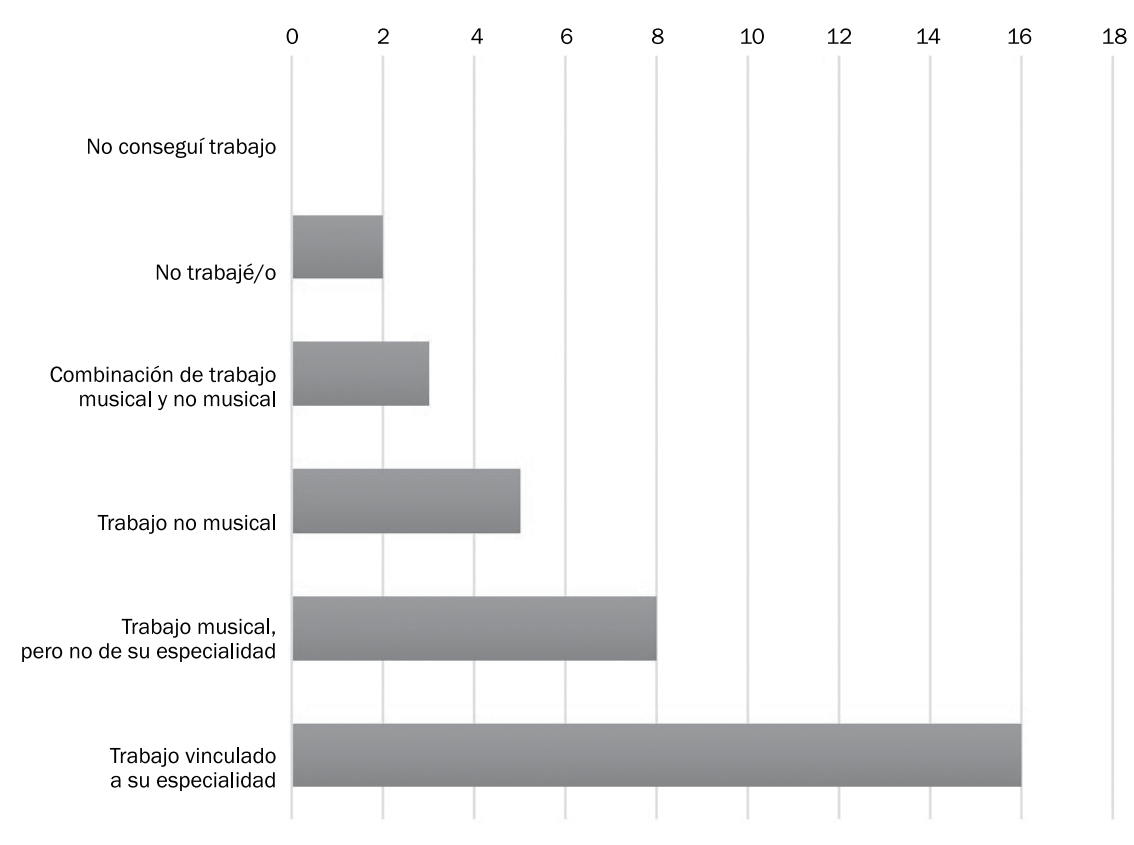

Gráfico 5: Tipos de trabajo que el encuestado ha tenido 
En cuanto a los empleos que han desarrollado, se dio a conocer que el $89 \%$ trabaja en un ámbito vinculado con su especialidad, el $44 \%$ trabaja en la música, pero no específicamente en su campo disciplinar, el $27 \%$ se desempeña en un empleo no musical, el i6\% combina empleos musicales con no musicales, mientras que el II\% no trabaja.

En resumen, el «pluriempleo» o lo que García Trabucco y otros (20II) llaman "carrera portafolio», es decir combinar «varios empleos simultáneos o sucesivos, en general de corta duración o tiempo parcial», es una es una realidad que no es ajena a los egresados y estudiantes de la Licenciatura en Flauta. A pesar de ello, vale la pena destacar que, según la investigación de Tomassiello (20IO-20I4), esta situación se refleja en todas las Carreras de la Facultad de Artes y Diseño de la Uncuyo.

\subsection{4 · Relación formación profesional - inserción laboral}

En el cuarto eje, como anticipa el título, se indagó sobre la relación que existe entre la formación brindada y la inserción laboral.

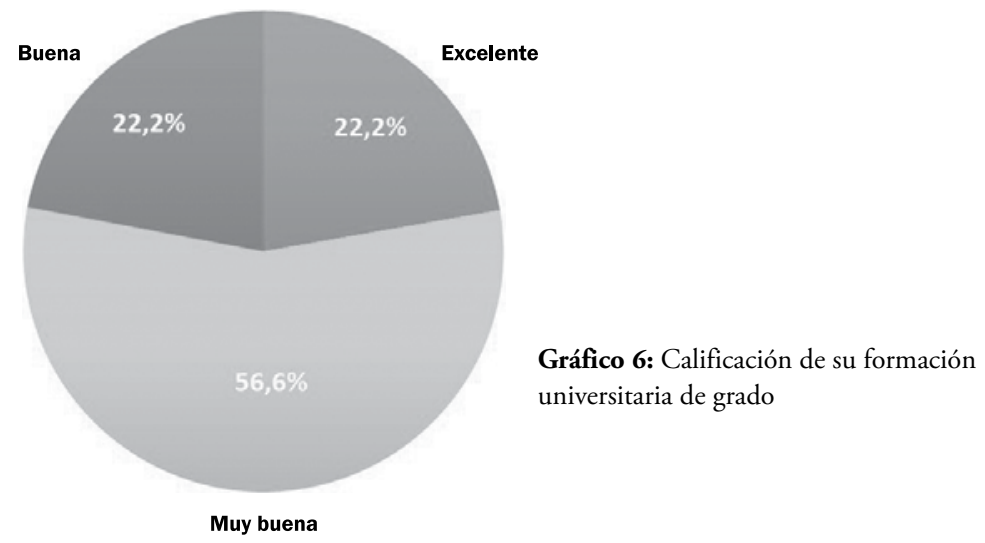




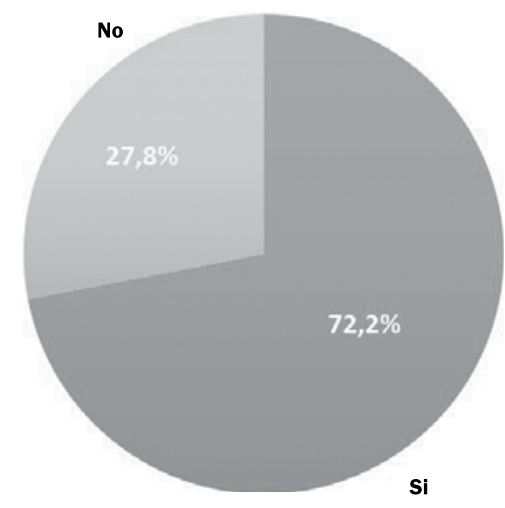

Gráfico 7: Distribución de respuesta en torno a la pregunta: ¿debió desarrollar otras habilidades, capacidades $y / o$ herramientas de las adquiridas en su formación de grado, para adquirir un puesto laboral?

De los resultados se pudo inferir que, a pesar de que los conocimientos adquiridos durante la formación de grado son percibidos como muy útiles, sería muy necesario ampliar el abanico de saberes y de prácticas para trabajar como flautista profesional, de acuerdo con el acotado mercado laboral.

\section{Perfil del egresado}

Y finalmente, en este último eje se buscó saber cuál es la percepción que tiene el egresado de su propio perfil profesional. Se les pidió que indicaran cuál de estas tres competencias, descriptas en el Perfil del Egresado del programa de la carrera, habían adquirido en mayor medida a lo largo de su formación de grado.

Ante esto, el Ioo\% declaró que pudo obtener la capacidad de interpretar en la flauta diversos géneros y estilos musicales, tanto en forma solista como en conjuntos; el $44,4 \%$, adquirió herramientas necesarias para realizar estudios, investigaciones y asesoramientos relacionados a su ámbito profesional específico; y, en último lugar, el 27,8\% pudo llevar adelante la planificación, conducción y supervisión de proyectos musicales. Cabe destacar, y hacer la salvedad, que existieron y existen espacios para iniciarse en la investigación tanto dentro de la Cátedra de 


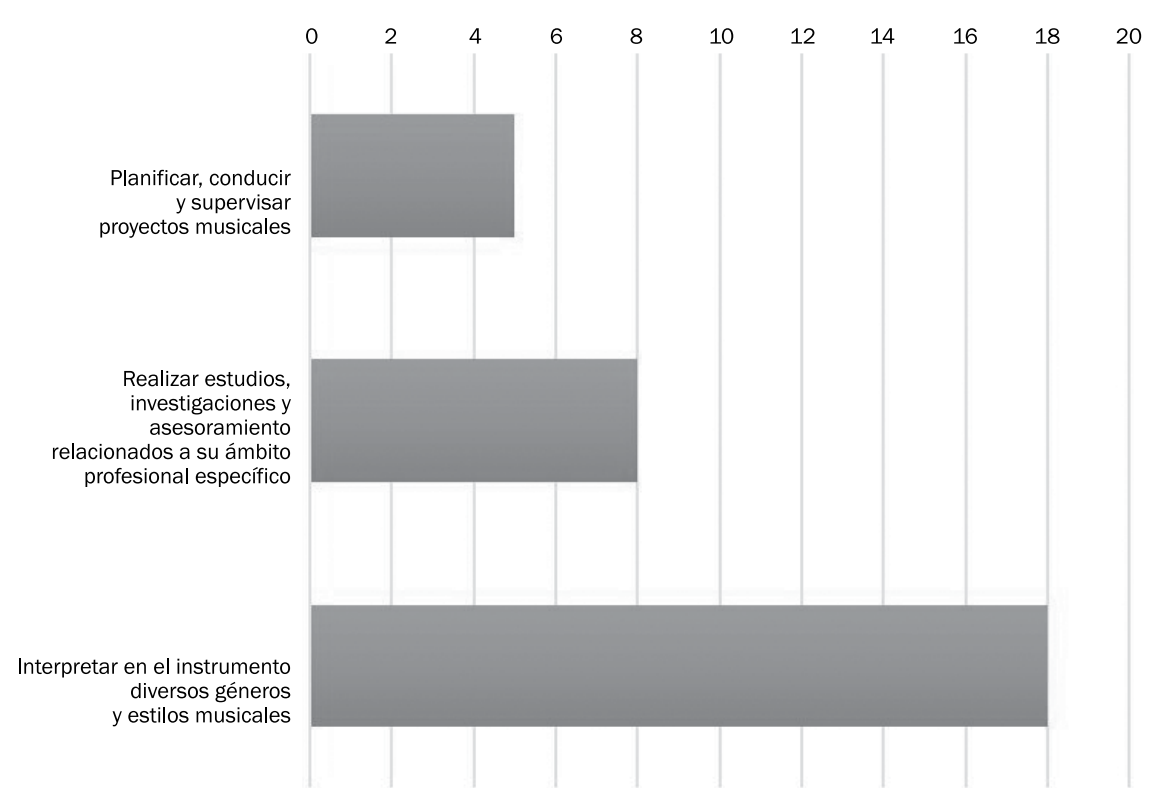

Gráfico 8: Percepción de su propio perfil de egresado

Flauta como en la Facultad de Artes y Diseño. Por ejemplo, becas o cursos/talleres de extensión que no siempre son aprovechados por el alumnado, ya sea por falta de difusión de los mismos o falta de interés o de tiempo de los estudiantes.

\section{2 - La realidad laboral actual del flautista profesional en la Argentina}

En las encuestas, los egresados y estudiantes avanzados expusieron sus expectativas laborales, las cuales estaban vinculadas directamente al terreno de la performance musical. A raíz de esto, surgió la necesidad de realizar un diagnóstico de la realidad laboral actual del flautista profesional en la Argentina, el cual se dividió en dos etapas.

En la primera, se llevó a cabo un relevamiento de la cantidad de Orquestas Sinfónicas, Bandas y Agrupaciones de Cámara Profesionales Argentinas que poseen cargos de flauta en sus filas. Se las discriminó por 
provincias, describió y, posteriormente, clasificó. Cabe destacar que el relevamiento se realizó a partir de los datos informados por los propios organismos en sus diferentes sitios webs o redes sociales, siendo esto un impedimento para obtener la información de todos los organismos sinfónicos del país, ya que muchos no cuentan con estos medios informáticos, lo cual imposibilita su divulgación. Y en una segunda etapa, se indagó sobre la carrera de interprete solista en Argentina.

\subsubsection{Clasificación de los organismos}

Se relevaron un total de 54 organismos y I54 puestos de flautas en ellos, los cuales no siempre son full time. Las orquestas fueron clasificadas teniendo en cuenta diferentes variables: nivel de profesionalismo, carga horaria que demanda, haberes percibidos, nivel de dependencia y forma de ingreso, resultando las siguientes clasificaciones:

Orquestas Sinfónicas Profesionales con dedicación exclusiva ${ }^{1}$ : Los músicos perciben haberes fijos. Su ingreso consiste en un Concurso Público y Abierto con etapas de audición de oposición y antecedentes. 16 organismos se enmarcan en esta categoría.

Orquestas Sinfónicas Profesionales con dedicación semiexclusiva ${ }^{2}$ : Los músicos perciben haberes fijos. El ingreso es igual que las orquestas con dedicación exclusiva. 7 organismos se enmarcan en esta categoría. Orquestas Semiprofesionales: Sus haberes, generalmente, son en concepto de beca con una duración determinada. Su ingreso presenta un grado de exigencia menor, no tan estandarizado y, por lo general, se convocan bajo el título de «audición». 6 organismos cumplen con estos requisitos:

1 Terminología usada en paralelismo con el Estatuto Universitario UNCuyo. Entendiendo la dedicación exclusiva, como un trabajo de tiempo completo con haberes que le permitan desarrollar su vida sin necesidad de buscar otro empleo. 2 Se asocia la dedicación semi-exclusiva con un trabajo de medio tiempo y en el que el trabajador debe valerse de otros ingresos para subsistir. 
Orquestas Sinfónicas Independientes o Autogestionadas: Los pagos, generalmente son por cachet, no hay sueldo fijo. Para ingresar a una Orquesta Independiente o Autogestionada, por lo general no se realiza audición, pero en caso de que se hiciera, solo se solicita una obra a elección o impuesta, y lectura a primera vista. Esta categoría cuenta con 5 organismos.

Orquestas Sinfónicas de las Fuerzas Armadas: Se detallaron las 4 más relevantes a nivel nacional, ya que cada fuerza cuenta con una banda en cada una de sus delegaciones a lo largo y ancho del país. Se percibe un haber fijo y en blanco. Su ingreso sigue un modelo estandarizado de audición teórica-práctica: una parte instrumental y otra de Teoría Musical.

En el caso de las Bandas Sinfónicas y Agrupaciones de Cámara, se tomó en cuenta su encuadre institucional, dividiéndolas de la siguiente manera: estatal (II) o privada (4). Su ingreso es similar al de una Orquesta Semi-Profesional.

\subsection{2 - Carrera de intérprete solista en Argentina}

Otra de las salidas laborales que habíamos mencionado para los intérpretes es la de Carrera de Solista. Para recabar información sobre esta posibilidad laboral, entrevistamos a tres flautistas profesionales, egresados de la Licenciatura en Flauta de la Uncuyo, con una trayectoria reconocida en este ámbito. Las preguntas apuntaban a conocer su formación profesional y sus comienzos como intérpretes solistas, la viabilidad de realizar una carrera como solista que permita hacer de ella un medio de vida, sus trabajos complementarios a esta carrera y las características que un flautista debía reunir para desenvolverse como solista.

Los tres entrevistados coincidieron en que sería casi imposible desarrollar una carrera sustentable como solista que permita hacer de ella un medio de vida, tanto en Argentina como en el mundo, porque no hay un mercado que lo posibilite. Uno de ellos citó el ejemplo de Emmanuel Pahud, considerado como un importante flautista actual, quien posee una trayectoria reconocida a nivel internacional como solista, aunque la complementa con un trabajo estable en la Filarmónica de Berlín. 
En cuanto a las características para ser solista, se requiere de una preparación técnica-musical y psicológica, acompañada por carisma, una personalidad fuerte y placer ante la exposición; habilidades que se adquieren con la práctica y la experiencia. En suma, vemos que, de acuerdo con la opinión de los entrevistados, la carrera de intérprete solista sería poco viable, a no ser que esté acompañada por otras instancias laborales.

\section{3 - Análisis de los Planes de Estudio de la Licenciatura en Flauta}

Para completar el panorama de la problemática planteada, se realizó una comparación entre los planes de estudio vigentes entre el período estudiado para así determinar si los cambios llevados a cabo en el nuevo plan mejoraron las posibilidades de inserción laboral de los egresados. Por ello, se procedió a realizar una observación estructurada de los planes de estudios de la Licenciatura en Flauta Ord. N ${ }^{\circ}$ 29/99-CS y 84/ıOCs, teniendo en cuenta las modificatorias, y se plasmaron en un cuadro comparativo de similitudes y diferencias bajo los siguientes parámetros: la duración de la carrera, el perfil y el alcance del título, la carga horaria total del Plan y los espacios curriculares con su respectiva carga horaria.

Luego, y con el fin de tener una mirada más amplia y real de la formación brindada por los planes, se pusieron en valor tanto las palabras de los egresados y estudiantes avanzados, expresadas en las encuestas realizadas, así como las de la Titular de la Cátedra de Flauta Mgter. Lic. Beatriz Plana, mediante una entrevista.

Finalmente, queremos aclarar que el análisis de los planes de estudio, en el contexto de este proyecto, fue solamente una observación estructurada para ver y comparar las herramientas que brinda cada plan. Somos conscientes de que un estudio más profundo de los planes de estudio podría constituirse en otra investigación, o sea, en una línea derivada de este proyecto. 


\section{$5 \cdot$ Conclusiones}

A la luz de la investigación realizada se observa que la temática es compleja de abordar debido a su multicausalidad, la cual genera responsabilidades compartidas entre las partes estudiadas, es decir el colectivo de egresados y estudiantes avanzados de la Licenciatura en Flauta, los planes de estudio, y el campo laboral. Por lo tanto, se le dará cierre a cada uno de los aspectos estudiados.

\section{$5.1 \cdot$ Expectativas laborales}

Por un lado, se observó una tensión entre las expectativas que se generan en los estudiantes durante su formación universitaria y las posibilidades reales de inserción laboral de los mismos. Como consecuencia, el egresado debe buscar otros trabajos para sustentarse, como por ejemplo la docencia musical, pudiendo implicar que éste enfrente sentimientos cercanos a la frustración si ésta no era su meta. El origen de este posible sentimiento de frustración puede relacionarse con el desencuentro entre las expectativas que tienen la mayoría de los estudiantes en relación con el desarrollo de una carrera performativa, sumado al peso que le da el Plan de Estudios a la interpretación, por un lado, y la realidad del acotado mercado laboral en el terreno de la performance musical, al cual el licenciado en flauta debe adecuarse, por otro.

Cabe destacar que durante la investigación fue puesto de manifiesto por los encuestados la falta de materias pedagógicas en el Plan de Estudios para desempeñarse como docentes en instituciones estatales y privadas. Sin embargo, la Universidad cuenta con un Ciclo de Profesorado para Licenciados en Instrumento, cuyo plan de estudios consiste en brindarles las herramientas pedagógicas necesarias para ejercer la docencia. Por lo tanto, esto no sería una falencia de la oferta académica de la Facultad de Artes y Diseño sino falta de información al respecto: muchos estudiantes desconocen este trayecto, o lo subestiman. 


\section{2 $\cdot$ Análisis del cambio del Plan de Estudio}

Buscando responder si el cambio de plan de estudio benefició la inserción laboral de los estudiantes, se determinó que no se presentaron diferencias significativas en cuanto a la inserción laboral de aquellos egresados del Plan aprobado en la Ordenanza $\mathrm{N}^{\circ} 29 / 99$-cs y aquellos que lo hicieron con el de la Ordenanza $\mathrm{N}^{\circ}$ 84/IO-Cs. No obstante, este dato debe mirarse desde una perspectiva más amplia porque, si bien a simple vista el agregado de disciplinas al Plan Nuevo no generó cambios en términos de mejoras de inserción laboral a corto plazo, sirvió, como bien explicó Plana, para «ampliarles (a los estudiantes y egresados) el panorama ideológico». Por ejemplo, este es el caso de Historia de la Música IV. Esta asignatura abre el abanico sobre diferentes caminos posibles para especializarse e insertarse laboralmente, por ejemplo, la Música Latinoamericana, ya sea para continuar una formación como intérprete o investigador. (Plana, B. entrevista, 2019).

\section{$5.3 \cdot$ Realidad laboral actual del flautista profesional en Argentina}

De acuerdo con el relevamiento realizado, se logró poner en conocimiento que la República Argentina cuenta con más de 50 organismos orquestales o de cámara, con diferentes niveles de profesionalismo, carga horaria, haberes percibidos y nivel de dependencia. A su vez, se relevaron más de 150 cargos de flautas dentro de estos organismos. No obstante, a pesar de no ser un número bajo, se observó que los puestos de trabajo para un flautista profesional en el terreno de la performance musical son escasos, habida cuenta de que solo el 39,1\% de los puestos recabados son de tiempo completo.

Es decir, la problemática radica en que continúan habiendo muchos más flautistas que puestos dentro de los organismos, ya que una vez que los cargos son cubiertos, el tiempo suele ser prolongado hasta que se genera una nueva vacante, debido a que esta depende de que el flautista se jubile, renuncie o alguna razón lo imposibilite a continuar en su 
cargo. Sumando la problemática de la pérdida de cargos, congelados o desfinanciados, que son suplantados por músicos eventuales, contratados por concierto y en carácter de refuerzo artístico, se aumenta la precariedad laboral y se produce el famoso «cuello de botella» en el ingreso a la profesión (Doñate, 20II).

Por otro lado, dando respuesta a la viabilidad de desarrollar una carrera sustentable como intérprete solista en la Argentina, se concluyó que se trata de una actividad que se complementa y se desarrolla paralelamente a otras, ya que sería imposible hacer de ella un único medio de vida. Habida cuenta de que es necesario desarrollar otras habilidades que van más allá de las adquiridas en la formación musical, las cuales son: poseer una preparación técnica-interpretativa y psicológica diferente a la usada habitualmente, acompańado de carisma, personalidad fuerte y placer ante la exposición.

\section{$5.4 \cdot$ Afirmación de la anticipación de sentido}

Luego del recorrido llevado a cabo en la investigación, se puede concluir que la anticipación de sentido apuntó en la dirección correcta dando respuesta a los interrogantes formulados. No obstante, el planteo puede enriquecerse de la siguiente manera: los egresados y estudiantes avanzados de la Licenciatura en Flauta de Carreras Musicales, FAD, uncuyo, perciben la necesidad de complementar las competencias que adquirieron en la formación de grado con otras herramientas que amplíen y diversifiquen su empleabilidad, en un contexto de escasas oportunidades laborales que además se encuentra en permanente cambio.

\section{$5.5 \cdot$ Herramientas}

Dichas herramientas, pueden dividirse en dos. Por un lado, las que los propios egresados y estudiantes avanzados de la Licenciados en Flauta deben procurar obtener de forma personal, las cuales son: el continuo 
perfeccionamiento instrumental que se prolonga más allá del estudio de grado, realizando clases magistrales, maestrías y/o doctorados en el país o en el extranjero; estudiar el ciclo de profesorado para Licenciados, ofrecido por la FAD - UNCuyo, para así obtener una formación pedagógica; desarrollar habilidades de autogestión, diseño y edición de material multimedia y producción en general, mediante cursos externos; generar una actitud positiva, receptiva y activa en la búsqueda de cursos y/o becas dentro de la Uncuyo, que fomenten la investigación en formato de actividades de extensión o de otras secretarías de la Universidad. En suma, tender a un criterio de capacitación constante y personalizada, de acuerdo a los propios intereses y posibilidades.

Por otro lado, se encuentran las herramientas brindadas por el Plan de estudio de la Licenciatura que no son suficientes a la hora de insertarse laboralmente. Las falencias detectadas son: el poco entrenamiento y/o experiencia orquestal real, la falta de materias que ayuden a aprender a auto-gestionarse como artista, la falta de difusión y de fomento de los cursos referidos a formación en la investigación, ya sean de extensión o de otras secretarías de la UNCuyo.

A su vez, se planteó el agregado de espacios curriculares, obligatorios u optativos, que le permitan al estudiante obtener una formación integral y en la que él pueda escoger el perfil que desee para su carrera laboral y profesional. Entre las mencionadas se planteó, el agregado de una asignatura de formación en prácticas socio-educativas, dado que una de las mayores salidas laborales en la actualidad, es la docencia en Orquestas Infanto-Juveniles que operan en territorio, para la cual no existe una formación específica; y espacios donde se les brinde la posibilidad de tener contacto con otras músicas para flauta donde se aborde, por ejemplo, la música antigua, contemporánea y latinoamericana.

\section{$5.6 \cdot$ Consideraciones finales}

No obstante, cabe destacar que la formación brindada por la Cátedra de Flauta, de la mano de los profesores Lars Nilsson y Beatriz Plana, es 
reconocida a nivel nacional e internacional por considerarse una de las Cátedras de Flauta más importantes, prestigiosas y de mayor nivel técnico-interpretativo, siendo un semillero del cual muchas orquestas de la Argentina y del mundo se nutren. Es decir, la formación flautística recibida es muy valorada en términos de brindar herramientas reales para la inserción laboral en el ámbito de la performance, convirtiéndose en una de las cátedras que más egresados insertó e inserta en el mundo laboral de las orquestas, bandas sinfónicas, conjuntos de cámara e interpretación solista.

Ante lo expuesto, a lo largo de la investigación se llegó a la conclusión de que, a pesar de que la Licenciatura en Flauta forma excelentes intérpretes, es necesario contar con planes de estudios más flexibles y con flautistas con mentes abiertas y un criterio de capacitación constante, para lograr así un enfoque que responda mejor a la realidad dinámica del mercado laboral donde puedan insertarse los Licenciados en Flauta. Es decir, abrir el abanico hacia nuevos horizontes laborales que esta época moderna y digitalizada posibilita. En palabras de la titular de la Cátedra de Flauta, uncuyo, «el mundo es dinámico y las demandas laborales son dinámicas, por eso debemos estar despiertos. Los estudiantes demandando, los profesores respondiendo, y la Universidad respaldando los cambios que haya que ir haciendo». (Beatriz Plana, entrevista, Io de junio, 2019) 


\section{REFERENCIAS BIBLIOGRÁFICAS}

BENNETT, DAWN (2010) La música clásica como profesión. Pasado, presente y estrategias para el futuro. Barcelona: Graó.

BÚJEZ, ALEJANDRo (2008). "La inserción laboral de los estudiantes de música: principales expectativas y salidas profesionales". Comunicación presentada en el I Congreso Internacional sobre Inserción Profesional a la Docencia. Sevilla, 25 de junio 2008.

CAPELLETTI, BEATRIZ; BYK, EDITH (2008) "Juventud y trabajo en la Argentina: diagnóstico y visión de los actores", en: Revista de trabajo. Año 4. № 6 , pp. 173-184. ISSN 0328-0764.

CONSEJO SUPERIOR (1999). "Ordenanza N 29/99-CS". Facultad de Artes y Diseño, Universidad Nacional de Cuyo. Mendoza, Argentina.

CONSEJO SUPERIOR (2010). "Ordenanza No 84/10-CS». Facultad de Artes y Diseño, Universidad Nacional de Cuyo. Mendoza, Argentina.

CUITIÑNO, CRISTINA (2016). «Inserción laboral de graduados de nivel superior: libro de memorias, jornadas de investigación: Universidad Nacional de Cuyo, 2015” Compilado por Cristina Cuitiño. - 1a ed. compendiada. - Mendoza: Universidad Nacional de Cuyo. Secretaría de Ciencia, Técnica y Posgrado.

DEIBE, ENRIQUe (2008). «Políticas de empleo para la inclusión», en: Revista de trabajo. Año 4. № 6, pp. 201-211. ISSN 0328-0764.

DIAZ, ESTHER (2007). Entre la tecnociencia y el deseo. La construcción de una epistemología ampliada. Buenos Aires: Biblos.

DIEZ, NANCY (2009-2014). "Análisis de los criterios de selección de músicos para integrar orquestas dependientes de organismos estatales argentinos: los casos de la Orquesta Sinfónica Nacional, la Orquesta Estable del Teatro Argentino de La Plata y la Orquesta Filarmónica de Buenos Aires durante el período 2009 - 2014». Tesis de Maestría en Administración del sector Cultural y Creativo: Orientación Artes Escénicas. Facultad de Ciencias Económicas, Universidad de Buenos Aires.

DOÑATE, LEANDRo (2011). "La Orquesta Sinfónica como agente empleador de los Licenciados en Instrumento: organización y relaciones laborales entre los organismos sinfónicos en Mendoza y sus integrantes». Tesina de Licenciatura en Oboe. Facultad de Artes y Diseño, Universidad Nacional de Cuyo. Mendoza. FACULTAD DE ARTES Y DiSEÑo (s/f). Licenciatura en Instrumentos. Recuperado el 15 de mayo de 2018, de http://fad.uncuyo.edu.ar/lic-en-instrumentosGARCíA TRABUCCO, ALEJANDRA; CUITIÑNO, CRISTINA (2009-2015). «Formación Profesional e inserción laboral de los egresados de carreras musicales Facultad de Artes y Diseño / UNCuyo. Etapas I, II y III!. Mendoza: UNCuyo. Secretaría de Ciencia, Técnica y Posgrado. 
GARCÍA, GLADYS; RIBA, ANA MARÍA; CAPUJ, SUSANA; FONZALIDA, CECILIA (2010). "La inserción laboral de los alumnos del profesorado de arte». Instituto 9-014 Profesorado de Arte, San Rafael, Mendoza.

GIBOIN, ALEJANDRO, GARCÍA TRABUCCO, ALEJANDRO Y CUITIÑO, CRISTINA (2012). «Problemática de la formación profesional e inserción laboral de los graduados de carreras musicales de la Facultad de Artes y Diseño, Universidad Nacional de Cuyo, Mendoza, Argentina". Ponencia presentada y publicada en las II Jornadas Nacionales sobre Estudios Regionales y Mercados de Trabajo. Red SIMEL - Universidad Nacional del Litoral. Santa Fe.

GIBOIN, ALEJANDRO (2014). "Inserción laboral de los/as artistas graduados/as de la Facultad de Artes y Diseño -Universidad Nacional de Cuyo- en el Gran Mendoza durante la última década. Primera etapa: músicos/as y artistas del espectáculo". III Jornadas Nacionales sobre estudios regionales y mercados de trabajo. Universidad Nacional de Jujuy, Facultad de Cs. Económicas y Unidad de Investigación en Comunicación, Cultura y Sociedad de la Facultad de Humanidades y Cs. Sociales, y Red SIMEL, San Salvador de Jujuy.

ISACOVICH, PAULA (2015). "Políticas para la inserción laboral de jóvenes: estudios en Latinoamérica y Argentina", en Revista Latinoamericana de Ciencias Sociales, Niñez y Juventud, volumen 13: N², pp. 893-905.

JACINTO, ClAUdia; WOLF, MARIELA, BESSEGA, CARLA; LONGO, MARIA EUGENIA (2004). "Jóvenes, precariedades y sentidos del trabajo". Ponencia presentada y publicada en el $7^{\circ}$ Congreso Nacional de Estudios del Trabajo. Asociación Argentina de Especialistas en Estudios del Trabajo. Buenos Aires.

PLANA, BEATRIZ (10 de junio, 2019). Comunicación personal: entrevista. Mendoza. PORRAS, MARÍA DEL CARMEN (2005). "Análisis comparativo de la inserción y desempeño laboral de los graduados de la carrera de Diseño Industrial, con las características psicológicas y de rendimiento académico relevadas en su trayectoria como estudiantes". Mendoza: UNCuyo. Secretaría de Ciencia, Técnica y Posgrado.

SOSA OVIES, MARTÍN (2012-2013). "La música como profesión: una aproximación desde el caso de la Escuela Universitaria de Música». Trabajo presentado en las XIII Jornadas de Investigación de la Facultad de Ciencias Sociales, UdelaR, Montevideo.

TAYLOR, STEVEN; BOGDAN, ROBERT (2000). Introducción a los métodos cualitativos de investigación. 3era. ed. Buenos Aires: Paidós. Recuperado el 12 de junio de 2018, de http://es.scribd.com/doc/78156817/Taylor-S-JBogdan-R-Introduccion-a-Los-Metodos-Cualitativos-de-Investigacion-OCR

TOMASSIELlo, ROBERTo (2010-2014). «Formación Profesional e Inserción Laboral en Artes, Diseño y Humanidades". Mendoza: UNCuyo. Secretaría de Ciencia, Técnica y Posgrado. 